\title{
Miniaturization of Superconducting Filters Using Hilbert Fractal Curves
}

\author{
Mario Barra, Carlos Collado, Member, IEEE, Jordi Mateu, Member, IEEE, and \\ Juan M. O'Callaghan, Senior Member, IEEE
}

\begin{abstract}
This work presents novel compact high-temperature superconductor microstrip resonators and filters based on the Hilbert space-filling fractal curve. Several Hilbert resonator configurations have been assessed by using a full-wave electromagnetic simulator and their miniaturization performance has been investigated, emphasizing the parameters which allow obtaining good tradeoff between compact size and losses. These resonators have proved to be useful for filter design. A four-pole $2.45-\mathbf{G H z}$ quasi-elliptic filter and a four-pole 1.9-GHz Chebyshev filter have been realized by patterning $10 \mathrm{~mm} \times 10 \mathrm{~mm} \mathrm{Y}_{2} \mathrm{Ba}_{3} \mathrm{Cu}_{7-\delta} \mathrm{O}$ thin films on $\mathrm{MgO}$ substrates. Insertion losses of the order of $0.2 \mathrm{~dB}$ have been measured, showing good agreement with the simulations.
\end{abstract}

Index Terms-Fractals, high-temperature superconductors, microstrip filters, microwave devices, mobile communications.

\section{INTRODUCTION}

$\mathbf{P}$ LANAR high-temperature superconductors (HTS) filters have been exhaustively studied during last decade since they may achieve good performance qualities comparable to those of heavy dielectric-loaded cavity filters.

To reduce the thin film HTS cost and relax the cooling requirements, miniaturization techniques for size reduction of planar filters are especially important in HTS filter design [1]. These techniques may include several ways of folding the transmission line in forms of loops [2], hairpins [3], spirals [4] or even fractal shapes [5], all of which have low RF losses despite their small size. In this work we explore the possibilities of using fractal Hilbert curves for the realization of HTS resonators and filters.

As a first step we assess the resonant frequency and unloaded quality factor of microstrip Hilbert shaped resonators versus the fractal iteration, and we compare them with conventional meander line resonators. Then, we propose several versions of the initial Hilbert resonator which are more suitable for the design

Manuscript received July 21, 2004; revised January 25, 2005. This paper was recommended by Associate Editor J. Mazierska. This work was supported in part by the Spanish Ministry of Science and Technology under Project MAT2002-04551-C03-03, and in part by the Generalitat de Catalunya (DURSI) under Grant 2001 SGR 00266. The work of M. Barra was supported by SCENET through an exchange visit grant.

M. Barra is with INFM-COHERENTIA and Dipartamento di Scienze Fisiche, Università dei Napoli "Federico II," Naples 80121, Italy (e-mail: mbarra@na.infn.it).

C. Collado and J. M. O'Callaghan are with the Universitat Politècnica de Catalunya (UPC), Campus Nord, Barcelona 08034, Spain (e-mail: collado@tsc.upc.es; joano@tsc.upc.es).

J. Mateu is with Centre Tecnològic de Telecomunicacions de Catalunya, Barcelona 08034, Spain (e-mail: jordi.mateu@cttc.es).

Digital Object Identifier 10.1109/TASC.2005.850553

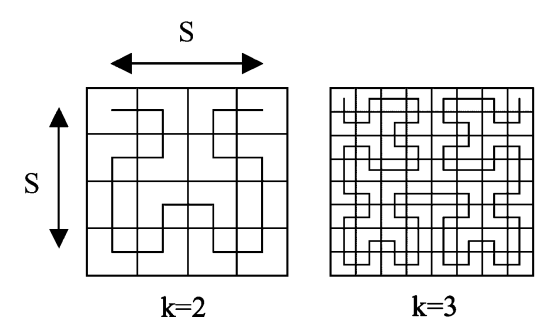

Fig. 1. Hilbert curves with second $(\mathrm{k}=2)$ and third $(k=3)$ iteration levels, respectively. The parameter $S$ is the length of the external side.

of filters. And finally, we have fabricated two four pole filters by using different resonators: a quasi-elliptic filter with central frequency $2.45 \mathrm{GHz}$ and $0.8 \%$ fractional bandwidth, and a Chebyshev filter centered to $1.9 \mathrm{GHz}$ with $2.6 \%$ fractional bandwidth. Each of them was patterned on a $10 \mathrm{~mm} \times 10 \mathrm{~mm}$ $\mathrm{Y}_{2} \mathrm{Ba}_{3} \mathrm{Cu}_{7-\delta} \mathrm{O}$ (YBCO) thin film on $\mathrm{MgO}$ substrate.

\section{HiLbert Microstrip RESONATORS}

The fractal Hilbert curve, as outlined in Fig. 1, consists in a continuous line which connects the centers of a uniform background grid. The fractal curve is fit in a square section of $S$ as external side.

By increasing the iteration level $k$ of the curve, one reduces the elemental grid size as $S /\left(2^{k}-1\right)$, the space between lines diminishes in the same proportion and the length of the curve increases as

$$
L(k)=\left(2^{k}+1\right) S .
$$

Ideally, one would like to increase the iteration of the Hilbert curve as much as possible in order to fit the resonator in the smallest area. However, there is a tradeoff between miniaturization (curves with high $k$ ) and quality factor $\left(Q_{0}\right)$ of a Hilbert resonator. For a microstrip resonator, the width of the strip $(w)$ and the spacing between the strips $(g)$ are the parameters which actually define this tradeoff [6]. Both dimensions ( $w$ and $g$ ) are connected with the external side $S$ and iteration level $k(k \geq 2)$ by

$$
S=2^{k}(w+g)-g .
$$

From this equation, it is clear that trying to obtain high miniaturization levels implies lower values of $w$ with the result that the dissipation losses increase and the quality factor diminishes. Note that $w$ almost halves from $k$ to $k+1$ if $S$ and the ratio $w / g$ is kept constant [6]. 
We show experimental results of a Hilbert resonator in Section II-B and comparisons between a Hilbert and a meander resonator in Section II-C.

\section{A. Hilbert Resonator Performance}

This subsection shows the resonant frequency $\left(f_{0}\right)$ and quality factor $\left(Q_{0}\right)$, obtained by using Momentum-Advanced Design System on several Hilbert microstrip resonators of YBCO on $\mathrm{MgO}$. The quality factor has been obtained from the transmission coefficient of the frequency response [2]. Besides to guarantee consistent comparison between several resonators, the meshing has been increased up to the point where no change is observed in their frequency responses.

A first set of resonators with a ratio $w / g$ equal to 1 is used to quantify the tradeoff between miniaturization and quality factor discussed previously, whereas a second set keeps $f_{0}$ constant and assesses the influence of $w / g$ on $Q_{0}$ and $S$. For all resonators, the parameters of the YBCO are taken from the data at $77 \mathrm{~K}$ of a commercial supplier, namely, surface resistance $5 f^{2} \mu \Omega$ with $f$ in $\mathrm{GHz}$ and London penetration depth $\lambda_{L}=$ $200 \mathrm{~nm}$. The material properties of the $\mathrm{MgO}$ are: relative dielectric constant $\varepsilon_{r}=9.6$, thickness $h=0.508 \mathrm{~mm}$ and loss tangent $10^{-6}$.

The first set consists on three series of microstrip Hilbert resonators ( $k=3, k=4$ and $k=5$ ) with $S$ values from $3 \mathrm{~mm}$ to $10 \mathrm{~mm}$. Fig. 2(a) shows the simulated $f_{0}$ versus $S$ for all series. As reference points for the achievable resonant frequencies in this range of dimensions, it can be observed that for $S=3 \mathrm{~mm}$ and $k=3, f_{0}$ is around $3.6 \mathrm{GHz}$, whereas for $S=10 \mathrm{~mm}$ and $k=5$, it is around $0.35 \mathrm{GHz}$.

For a given $S$, it can be observed that, although according to (1) the length of the strip almost doubles at each iteration-from $k$ to $k+1-$ the fundamental resonance frequency does not halve. This phenomenon is due to the couplings between the different turns of the Hilbert resonator which practically define an equivalent shorter path for the signal. Obviously this effect gets stronger with the increase of $k$, since a reduction of the interspacing among the turns takes place. In this way, for large values of $k$, the ratio $f_{0}(k+1) / f_{0}(k)$ between the resonant frequencies of two iterations tends to grow toward 1 . This means, from a practical point of view, that only iterations up to $k \leq 6$ of a Hilbert resonator guarantee an effective miniaturization improvement.

Fig. 2(b) plots the unloaded quality factor of this first set of resonators. The reduction in quality factor that occurs when $S$ is reduced is due to the increased loss due to a higher resonant frequency and a narrower line width. In a second part, we have focused our attention on a fixed frequency of $2 \mathrm{GHz}$ which results of interest for $3 \mathrm{G}$ wireless applications. We have evaluated how the $w / g$ ratio affects $Q_{0}$ and $S$.

Table I lists the width of the strip, the external side and the quality factor for $k=3,4,5$ and $w / g=0.5,1,1.5$. For every $w / g$ series, $S$ almost halves comparing $k=3$ with $k=5$, but $w$ is reduced from 8 to 10 times, which significantly lowers the quality factor. On the other hand, increasing $w / g$ and keeping $k$ fixed, $S$ slightly increases, making the resonator less miniaturized, and improving $Q_{0}$ since the microstrip width increases.

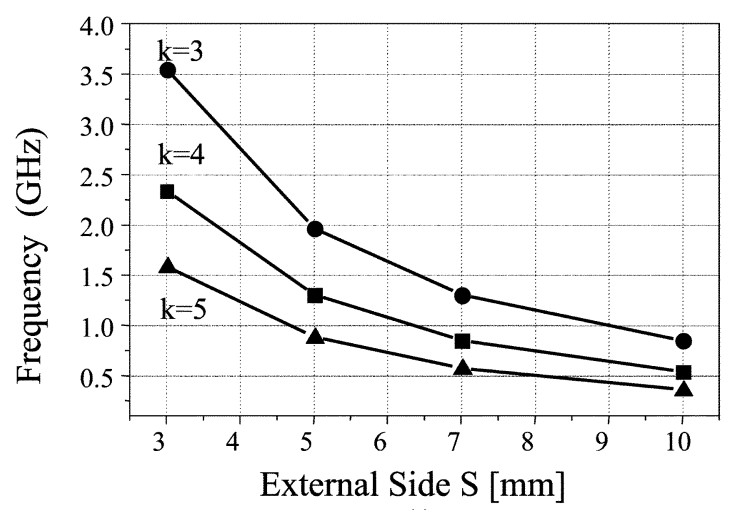

(a)

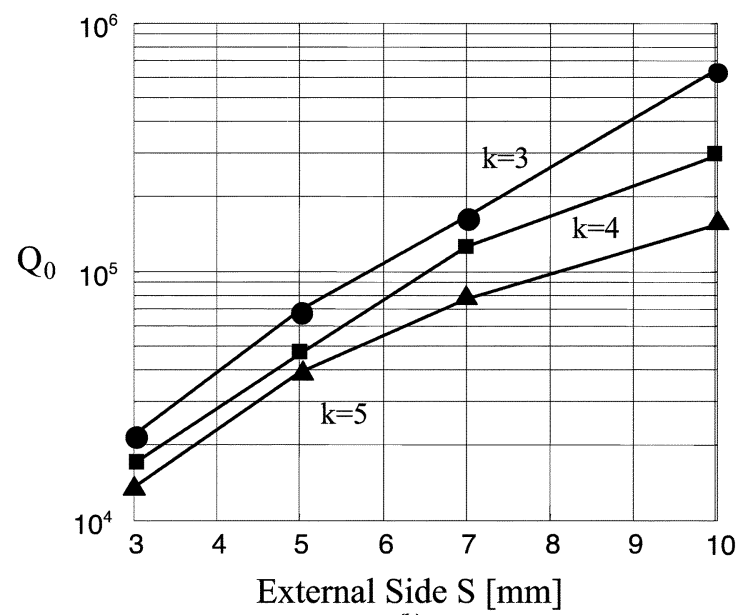

(b)

Fig. 2. (a) Fundamental resonant frequencies of Hilbert resonators with $k=3,4,5$ and (b) corresponding unloaded quality factor as a function of the external side $S$. Note that these quality factors are not all at the same frequency.

TABLE I

EXTERNAL SidES AND Microstrip WidTHS OF HiLBERT RESONATORS ON MGO WITH $\mathrm{K}=3,4$, AND 5 AT FREQUENCY $2 \mathrm{GHz}$

\begin{tabular}{l|c|c|r}
\hline \hline & $w(\mathrm{~mm})$ & $S(\mathrm{~mm})$ & \multicolumn{1}{|c}{$Q_{0}$} \\
\hline \hline$w / g=0.5, k=3$ & 0.2 & 4.60 & 56,100 \\
\hline$w / g=0.5, k=4$ & 0.069 & 3.33 & 18,500 \\
\hline$w / g=0.5, k=5$ & 0.024 & 2.35 & 6,800 \\
\hline$w / g=1, k=3$ & 0.330 & 4.95 & 64,200 \\
\hline$w / g=1, k=4$ & 0.115 & 3.58 & 24,800 \\
\hline$w / g=1, k=5$ & 0.041 & 2.58 & 8,700 \\
\hline$w / g=1.5, k=3$ & 0.4 & 5.09 & 67,700 \\
\hline$w / g=1.5, k=4$ & 0.142 & 3.71 & 27,900 \\
\hline$w / g=1.5, k=5$ & 0.051 & 2.71 & 10,400 \\
\hline \hline
\end{tabular}

By combining the results from Fig. 2 and the ones outlined in Table I one may identify the maximum miniaturization level that one could achieve if a minimum $Q_{0}$ was required at a given frequency.

The Hilbert resonator with $k=4$ and $w / g=1$ gives a good trade-off between miniaturization level and $Q_{0}$ at $2 \mathrm{GHz}$. In short, the quality factor is around 25000 and the external dimension $S$ is only $3.58 \mathrm{~mm}$, that is $0.06 \lambda_{g}$, where $\lambda_{g}$ is the wavelength at $2 \mathrm{GHz}$ for a $50 \Omega$ microstrip line on $\mathrm{MgO}$.

\section{B. Measurements of a Single HTS Hilbert Resonator}

The resonator of Table I with $k=4$ and $w / g=1$ [Fig. 3(a)] was fabricated from thin-film YBCO (700 nm thick) on a 


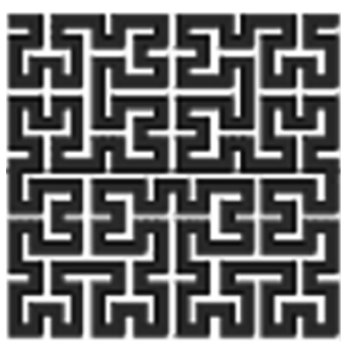

a)

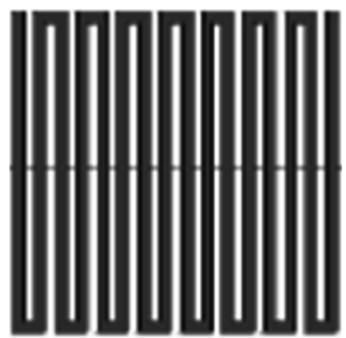

b)
Fig. 3. (a) Hilbert resonator with $k=4, w / g=1$ and external side $3.58 \mathrm{~mm}$ and (b) equivalent meander resonator.

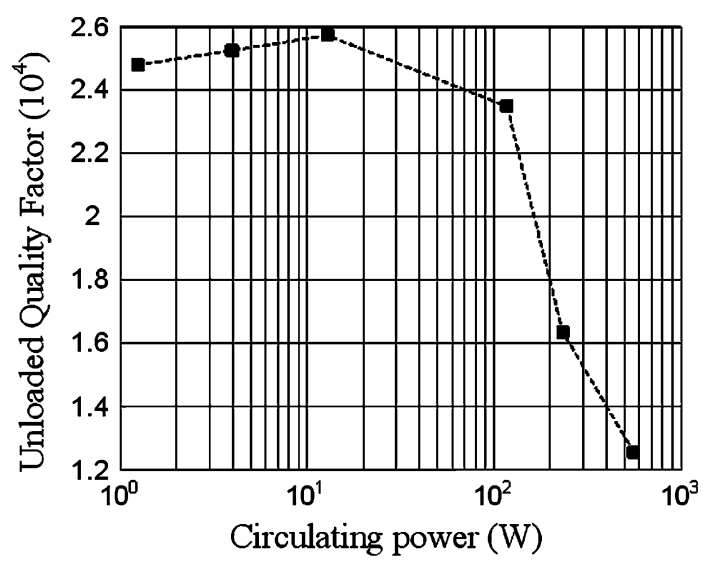

Fig. 4. Measured unloaded quality factor as a function of the circulating power in the resonator of Fig. 3(a).

$10 \times 10 \mathrm{~mm} \mathrm{MgO}$ substrate, using standard photolithographic processes. The resonator was measured at $77 \mathrm{~K}$ in a liquid nitrogen bath. The loaded resonant frequency was $1.84 \mathrm{GHz}$, $80 \mathrm{MHz}$ lower than the expected value from simulations, and the measured $Q_{0}$ was 26000 , which is in good agreement with simulations (see Table I). This value is similar to those reported in very recent papers for other compact resonators [3], [4] and no degradation of the frequency response was observed at circulating power levels up to $20 \mathrm{~W}$ [7]. To illustrate this last statement, Fig. 4 shows the measured unloaded quality factor as a function of the circulating power.

\section{Hilbert Versus Meander Resonator}

We have compared electromagnetic simulations of the resonator of Fig. 3(a) with those of its meander line counterpart [Fig. 3(b)], since the meander line achieves the same length for the same strip width, gap, and external side. The simulations reveal that the meander line has the same quality factor and resonates at almost $30 \mathrm{MHz}$ higher frequency than the Hilbert one, which confirms the results of [8]. It can be explained because the coupling among the turns is higher in a meander resonator due to its longer confronted length, so the equivalent reduction of the signal path is slightly stronger than that produced in the Hilbert device.

This higher coupling between turns also makes the electromagnetic behavior more dependent on the tolerances of physical parameters like the substrate thickness $h$ [9]. Fig. 5 shows the shift on the resonant frequency for these resonators versus $h$. For a change of $h$ around $\pm 5 \%$, the frequency shift of the

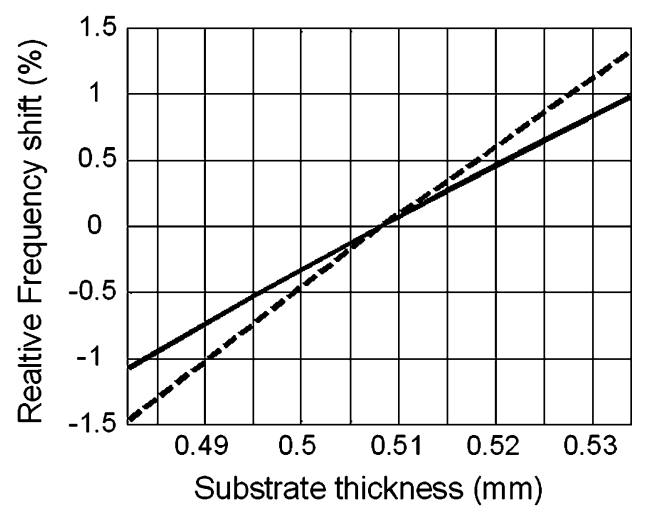

Fig. 5. Comparison between the relative resonant frequency shift of the Hilbert resonator (continuous line) and the equivalent meander resonator (dashed line) as a function of the thickness substrate.

Hilbert resonator is $\pm 1 \%$, whereas for the meander line resonator is $\pm 1.5 \%$. As discussed in [9], such differences change the resonant frequency of the filter resonators and may spoil the filter response.

We can conclude that neither the miniaturization capability nor the sensitivity to tolerances in material or substrate properties are very different between both resonators. However, and as shown in Sections III-A and III-B, the self-similarity property of the Hilbert curve, offers more flexibility than the meander curve to develop resonators with adequate coupling properties for the design of filters. In other words, with an elemental section of the Hilbert curve, i.e., for a given width, gap and lengths of the turns of the strips, one may change the field distribution by combining those elemental sections. Note that this is convenient since the field distribution has strong effect in the coupling between resonators and therefore in the filter topology.

\section{FILTER DESIGN AND EXPERIMENTAL RESUlTS}

We have fabricated two filters with central frequencies around $2 \mathrm{GHz}$, both of which fitted in a $10 \mathrm{~mm} \times 10 \mathrm{~mm}$ area. These filters contain resonators which are versions of the basic Hilbert topology of Fig. 3(a). These variations are introduced to favor the proper coupling among the various resonators and the coupling to the input and output feed lines.

\section{A. Quasi-Elliptic Filter}

A quasi-elliptic response is characterized by having transmission zeros close to the edge of the passband. These zeros may be obtained by cross-coupling a pair of nonadjacent resonators in such a way that the sign of the cross-coupling is opposite to that of the sequential couplings [9]. This can be achieved by the combination of electric coupling and magnetic coupling between resonators. This is not easily obtained in the basic topology of Fig. 3(a), since at resonance, the current (magnetic field) maximum is located at the centre of the layout and there are two electric field maxima, one at each end of the folded line.

Fig. 6 shows the modified Hilbert topology, where a capacitive load has been added by lengthening and coupling the open ends of the line to obtain a single point where the electric field is maximal. Also, the mid point of the folded line has been brought to the periphery of the resonator layout by rearranging two sections of the folded line. These two sections (which are Hilbert 


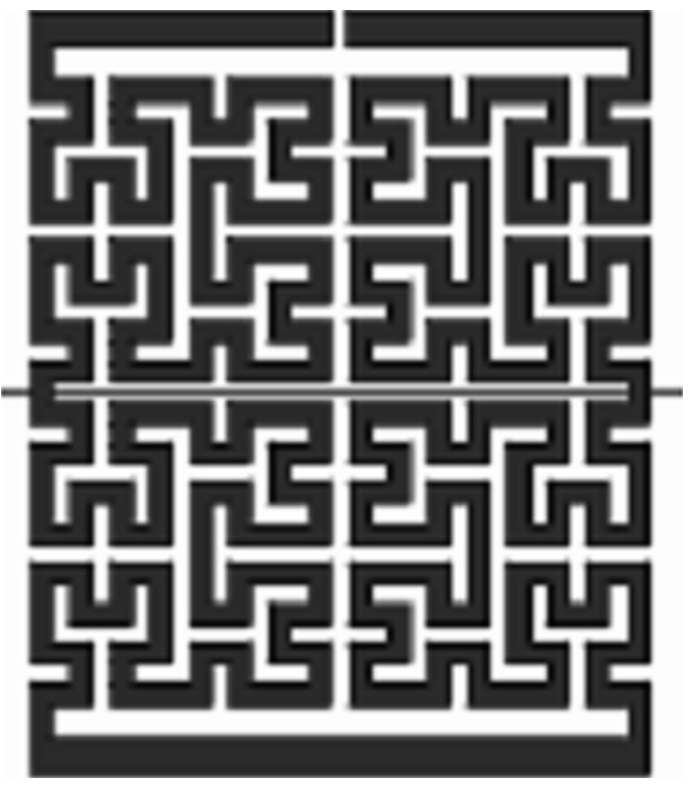

Fig. 6. Hilbert resonator with capacitive load and accessible mid point of folded line (current maximum).

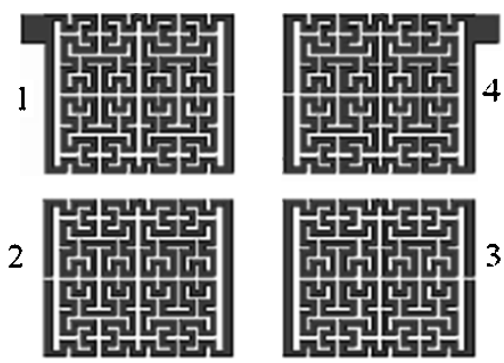

Fig. 7. Quasi-elliptic four pole filter layout.

curves with $k=3$ ) take the half lower part of the layout in Fig. 3(a). By cutting and rotating them and adding a straight line at the lower side of the resonator, one can convert Fig. 3(a) into Fig. 6.

This variation of the original Hilbert curve has been tested for the design of quasi-elliptic filters with different bandwidths. The coupling between resonators versus distance has been obtained following the procedure described in [9]. As shown in the upper part of Fig. 7, input and output lines can be tapped directly to the resonator. The external quality factor $\left(Q_{\text {ext }}\right)$ and the filter bandwidth are set by the distance between the tap point and the midpoint of the straight side in the modified resonator. With this arrangement we can obtain fractional bandwidths between $0.5 \%$ and $3 \%$.

Using the topology of Fig. 7, we have designed a four pole quasi-elliptic filter centered at $f_{0}=2.45 \mathrm{GHz}$ with $20 \mathrm{MHz}$ bandwidth following the synthesis procedure detailed in [9]. The coupling coefficients are $k_{12}=0.00725, k_{23}=0.00639, k_{14}=-0.00142$ and the external quality factor is $Q_{\text {ext }}=114.6$.

The filter was fabricated on a $10 \times 10 \mathrm{~mm}$ YBCO thin film on $\mathrm{MgO}$. The dimensions of the basic resonator were $2.68 \times 3.27 \mathrm{~mm}$ with a strip width of $90 \mu \mathrm{m}$, and the whole filter occupied an area of $7.9 \mathrm{~mm} \times 6.2 \mathrm{~mm}$.
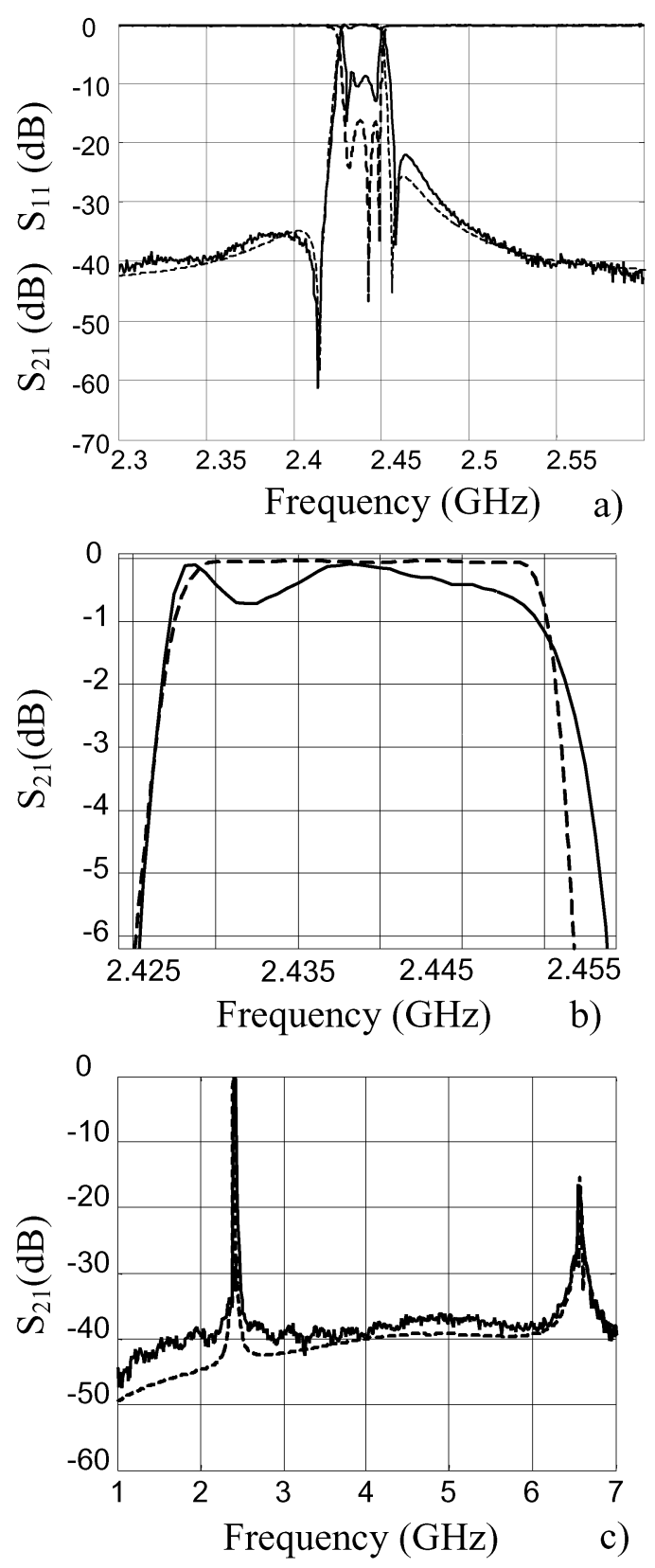

Fig. 8. (a) Comparison between measured (continuous line) and simulation (dashed line) responses of the four pole quasi-elliptic filter. In-band (b) and wideband (c) responses.

The filter was tested in a liquid nitrogen bath up to $0 \mathrm{dBm}$ input power. Fig. 8 shows both the simulated and measured response without tuning. In this figure, the frequency scale of the simulated response has been shifted to match the simulated one, since there was a little discrepancy in the central frequency $\left(f_{0}\right)$ of both responses $\left(f_{0}=2.450 \mathrm{GHz}\right.$ in the simulated response versus $2.438 \mathrm{GHz}$ in the measured one).

Minimum insertion losses are about $0.2 \mathrm{~dB}$, which is in good agreement with simulation data [Fig. 8(b)], but the in band response seems to suffer a ripple distortion (maximum ripple of $0.6 \mathrm{~dB}$ and minimum return loss of $8 \mathrm{~dB}$ ) due a little detuning condition.

Fig. 8(c) also shows good agreement for the wideband filter response. The absence of the second harmonic response is sig- 


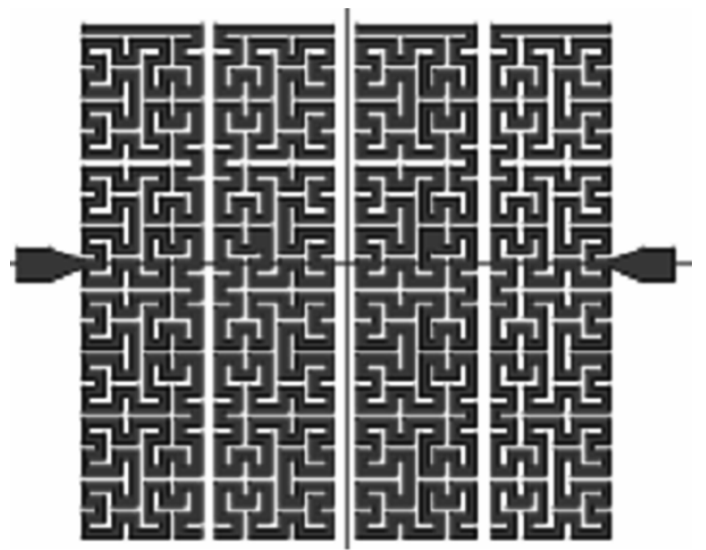

Fig. 9. Chebyshev four-pole filter layout.

nificant, and is a consequence of the electric and magnetic field distribution on the resonator for the first and second modes. For the first mode, the maximum of the magnetic field is reached at the continuous straight section (lowest part in Fig. 6), allowing good coupling between the lower resonators of Fig. 7. In contrast, the maximum magnetic field in the second mode occurs in the inner part of the resonator, resulting in a very weak coupling between the resonators.

\section{B. Chebyshev Filter}

In contrast with quasi-elliptic topologies, Chebyshev filters are defined as a ladder network with only sequential couplings [9]. It is therefore obvious that a four pole Chebyshev filter with $2 \mathrm{GHz}$ central frequency would not fit in a $10 \mathrm{~mm} \times 10 \mathrm{~mm}$ thin film on $\mathrm{MgO}$ substrate by using any of the resonators presented so far.

As done in the quasi-elliptic case, we propose an alternative version of the initial $k=4$ Hilbert curve. In this case, we have changed the orientation and disposition of the $k=3$ Hilbert curves, rearranging them in a vertical sequence. This new resonator is shown in Fig. 9. Its dimensions are $1.83 \mathrm{~mm}$ wide and $7.91 \mathrm{~mm}$ high with a microstrip width of $120 \mu \mathrm{m}$.

With this topology, a four pole $50 \mathrm{MHz}$ bandwidth Chebyshev filter centered to $f_{0}=1.95 \mathrm{GHz}$ has been designed occupying an area of $8.2 \mathrm{~mm} \times 7.93 \mathrm{~mm}$ (Fig. 9). In this case, the resonators can be placed very close to each other while keeping a low degree of coupling among them. In short, the space between resonators of Fig. 9 is lower than $0.3 \mathrm{~mm}$ and coupling coefficients of the order of 0.001 -needed for bandwidths smaller than $0.25 \%$ - could be obtained with distances typically about $1 \mathrm{~mm}$. Note that every resonator is provided with a final straight termination. Its length varies the distance between the center tap point and the point of maximum current in the line, which is used to adjust the $Q_{\text {ext }}$.

The filter was fabricated and was measured at $65 \mathrm{~K}$ in a closed cycle cryogenic system. As shown in Fig. 10, the measured response was centered at $1.9 \mathrm{GHz}$ so that a discrepancy of $50 \mathrm{MHz}$ was found between measurements and predictions (the frequency scale of the simulated data has been shifted to compare both traces). The $3 \mathrm{~dB}$-bandwidth [Fig. 10(b)] was also $10 \mathrm{MHz}$ lower than expected. Factors such as a non uniform value of the permittivity or of the substrate thickness may have influenced in the filter performance since it occupied
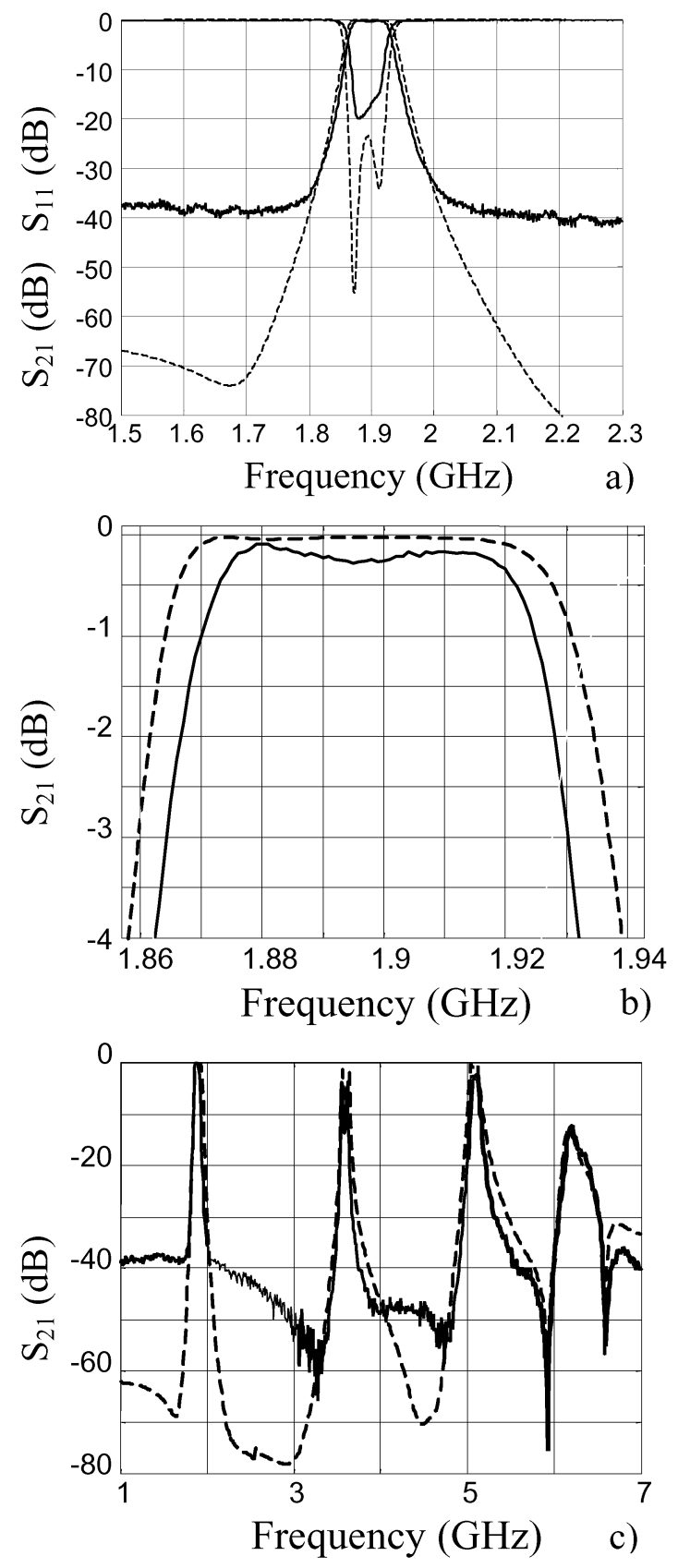

Fig. 10. (a) Chebychev filter performances, comparison between measurements at $\mathrm{T}=65 \mathrm{~K}$, (continuous line) and simulation (dashed line), (b) in-band, and (c) wideband responses.

almost all the thin film area. Moreover, the simulators have not considered the effect of the reduced dimensions of the metallic box which certainly has limited also the out band rejection value (about $40 \mathrm{~dB}$ ) with a behavior very similar to that described in [4]. The in-band performances in terms of insertion losses $(0.1 \mathrm{~dB}$ as minimum value $)$ and ripple $(0.2 \mathrm{~dB}$ as maximum value) were very good, demonstrating again the good tradeoff between quality factor and miniaturization that this kind of resonators can offer.

Fig. 10(c) shows the wideband filter response from $1 \mathrm{GHz}$ to $7 \mathrm{GHz}$ with a strong spurious response at each harmonic of the fundamental frequency. As done in the quasi-elliptic configuration, this can be explained from the electric and magnetic field 
distribution of the resonant modes. The magnetic and electric field maxima for the fundamental and second resonances are confronted between sequential resonators, so that the strength of the couplings does not change significantly from the first and second mode. This evidence suggests the possibility to design filters with multiple passbands taking advantage of the geometrical properties of the fractal Hilbert curve.

\section{Summary AND CONCLUSIONS}

In this work the miniaturization performances of a novel type of HTS microstrip resonator based on the Hilbert curves have been investigated. The fractal structure was analyzed considering several levels of iteration and putting in evidence the effect of the different geometrical parameters on the obtainable miniaturization level and quality factors. The performance in terms of quality factor of a single Hilbert $2 \mathrm{GHz}$ resonator with a side of $3.58 \mathrm{~mm}$, has been tested at $77 \mathrm{~K}$ yielding a quality factor of about 26000 . Quasi-elliptic and Chebyshev filters have been realized, showing the flexibility of the Hilbert curve to design resonators with good coupling properties. Both filters have been fabricated in $10 \mathrm{~mm} \times 10 \mathrm{~mm}$ double sided $700 \mathrm{~nm}$ YBCO thin films on $0.508 \mathrm{~mm}$ thick $\mathrm{MgO}$ substrate. The measured minimum insertion losses $(0.1 \mathrm{~dB}-0.2 \mathrm{~dB})$ confirm the good tradeoff between quality factor and reduced dimensions. For both filters, discrepancies between measured and simulated $f_{0}$ and bandwidth have been evidenced, resulting object of future investigations.

\section{ACKNOWLEDGMENT}

The authors wish to thank the extensive technical work provided by L. Enrique in the processing of samples. They also thank J. Romeu, J. M. González and A. Cassinese for the stimulating discussions.

\section{REFERENCES}

[1] M. J. Lancaster, F. Huang, A. Porch, B. Avenhaus, J.-S. Hong, and D. Hung, "Miniature superconducting filters," IEEE Trans. Microw. Theory Tech., vol. 44, no. 7, pp. 1339-1343, Jul. 1996.

[2] J.-S. Hong and M. J. Lancaster, "Couplings of microstrip square openloop resonators for cross-coupled planar microwave filters," IEEE Trans. Microw. Theory Tech., vol. 44, no. 11, pp. 2099-2109, Nov. 1996.

[3] G. L. Matthaei, "Narrow band, fixed tuned and tunable bandpass filters with zig-zag hairpin comb resonators," IEEE Trans. Microw. Theory Tech., vol. 51, no. 4, pp. 1214-1219, Apr. 2003.

[4] F. Huang, "Ultra-compact superconducting narrow band filters using single and twin spiral resonators," IEEE Trans. Microw. Theory Tech. vol. 51, no. 2, pp. 487-491, Feb. 2003.

[5] J. M. O'Callaghan, C. Puente, N. Duffo, C. Collado, and E. Rozan, "Fractal and space-filling transmission lines, resonators, filters and passive network elements," Patent-WO0 154221.

[6] M. Barra, C. Collado, J. Mateu, and J. M. O'Callaghan, "Hilbert fractal curves for HTS miniaturized filters," in Microwave Symp. Dig., 2004 IEEE MTT-S Int., pp. 123-126.

[7] H. Chaloupka, "Microwave applications of high-temperature-superconductors," in Applications of Superconductivity, H. Weinstock, Ed. Norwell, MA: Kluwer, 2000, pp. 295-383.

[8] S. R. Best, "A comparison of the performance properties of the Hilbert curve fractal and meander line monopole antennas," Microw. Opt. Technol. Lett., vol. 35, no. 4, pp. 258-262, Nov. 2002.

[9] J. S. Hong and M. J. Lancaster, Microstrip Filters for RF/Microwave Applications. New York: Wiley, 2001.

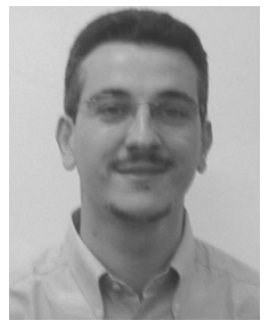

luminescent devices.
Mario Barra was born in Naples, Italy, on June 30, 1973. He received the Electronic Engineering degree and the Ph.D. degree from the from the Università di Federico II, Naples, Italy, in 2000 and 2004, respectively. From March to September 2003, he was a visiting student at Universitat Politecnica de Catalunya, in Barcelona, Spain. His present primary research interests include microwave superconducting devices, characterization in D.C. and R.F. range of the electronic transport properties of innovative materials for electronics, fabrication and testing of organic electro-

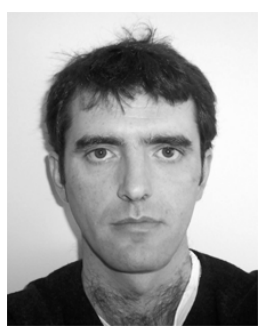

Carlos Collado (M'03) was born in Barcelona, Spain, in 1969. He received the Telecommunication Engineering degree and the Ph.D. degree from the Universitat Politècnica de Catalunya (UPC), Barcelona, Spain, in 1995 and 2001, respectively. He received the Master in Bioengineering degree from the Centre de Recerca en Enginyeria Biomèdica, UPC, in 2002.

Since 1998, he has been a Professor at UPC, where he has been teaching courses on theory of electromagnetism, microwave laboratory, and high-frequency devices and systems. His primary research interests include microwave devices and systems, electrooptics applications, and superconducting devices.

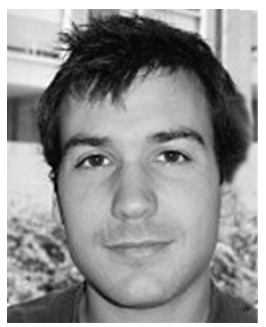

Jordi Mateu (M'03) was born in Llardecans, Spain, in 1975. He received the Telecommunication Engineering degree and the Ph.D. degree from the Technical University of Catalonia (UPC), Barcelona, Spain, in 1999 and 2003, respectively.

In September 1999, he joined UPC's Electromagnetic and Photonic Engineering Group (EEF) as a graduate student where he was granted a scholarship for the pursuit of his Ph.D. degree. From July to October 2001, he held a visiting research appointment at Superconductor Technologies, Inc., (STI), Santa Barbara, CA. In September 2004, he was visiting Postdoctoral Researcher with the Electronics and Electrical Engineering Laboratory, National Institute of Standards and Technology (NIST), Boulder, CO. Since October 2002, he has been a Research Associate at CTTC's Communications Subsystems Area. He is also part-time Assistant Professor at Universitat Autònoma de Barcelona (UAB). His primary research interests include microwave and photonic devices and systems, superconducting devices, characterization of nonlinearities of high-temperature superconductive films, and study of efficient methods for the analysis of nonlinear effects in communication systems.

Dr. Mateu received the 2003 prize for the best Doctoral Thesis in Fundamental and Basic Technologies for Information and Communications and their Applications by COIT (Colegio Oficial de Ingenieros de Telecomunicación) and AEIT (Asociación Española de Ingenieros de Telecomunicación).

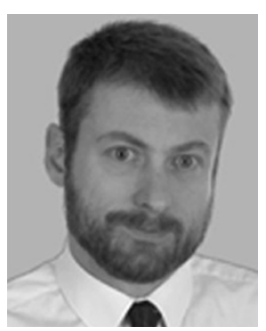

Juan M. O'Callaghan (M'84-SM'01) was born in Tortosa, Spain, in 1963. He received the telecommunication engineering degree from Universitat Politècnica de Catalunya (UPC), Barcelona, Spain, in 1987. He received the M.S. and Ph.D. degrees from the University of Wisconsin, Madison.

$\mathrm{He}$ was an Intern at Honeywell's Systems Research Center from July 1989 to December 1989. In 1993, he joined the faculty at UPC, where he has been teaching courses on radar, radio-navigation, and high-frequency devices and systems. He has been a Full Professor at UPC since May 2003. His research interests include microwave devices and systems, including high-frequency applications of superconductors. 